\title{
Paediatric Injectable Guidelines. 5th ed.
}

\section{Bruce Chio}

Pharmacist - Clinical leader

(surgical)

Hospital

Brisbane

\section{Aust Prescr 2017;40:158}

https://doi.org/10.18773/

austprescr.2017.049
Lady Cilento Children's

Lilley L, Legge D.

Parkville, Vic.: The Royal Children's Hospital

Melbourne; 2016.

90 pages

Also available online at www.rch.org.au/pig

The latest edition of this book contains 287 up-todate drug monographs. They provide advice on preparation and administration of injectable medications. The information is specific to the paediatric setting with details such as powder volume to calculate part vial

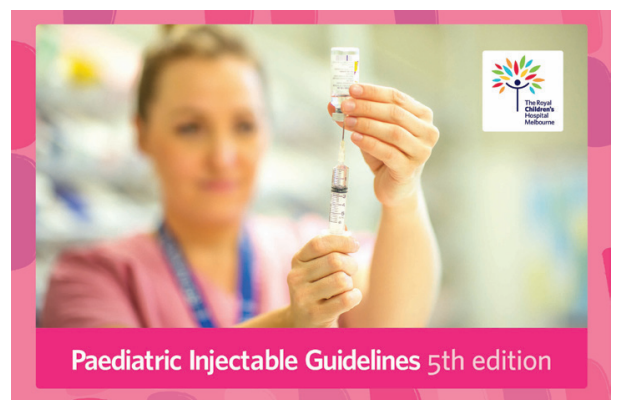
doses, maximum concentrations to reduce fluid volume, and line access considerations. Precautions around administration are also given without distracting the user with unnecessary detail.

Some monographs, however, lack specialised detail that is occasionally required in the paediatric setting. For example, dilution advice for subcutaneous injection of small doses of enoxaparin is not included but hopefully will be considered for future editions.

The Parenteral Nutrition and Fat Emulsion compatibility information is an extremely valuable section of the book. It provides advice on intravenous drugs which can and cannot be run concurrently via Y-site. Users should, however, interpret the information with caution to ensure their patient's parenteral formulation is comparable to The Royal Children's Hospital Melbourne preparations.

With safety features such as tall-man lettering, easy-to-read formatting and concisely written information, it is an essential resource to have in a busy environment. The book is physically robust. It is resilient to water and does not glare under bright lighting. These guidelines provide reliable, comprehensive and specific information for practitioners working in paediatric settings. 